\title{
Session II
}

\section{Observations of solar and stellar variability}

\author{
Chair: A. G. Kosovichev
}




\title{
One solar cycle of solar astrometry with $\mathrm{MDI} / \mathrm{SOHO}$
}

\author{
Marcelo Emilio ${ }^{1}$, Jeff R. Kuhn ${ }^{2}$ and Rock I. Bush ${ }^{3}$ \\ ${ }^{1}$ Observatório Astronômico-Departamento de Geociências Universidade Estadual de Ponta \\ Grossa, Paraná, Brazil \\ email:memilio@uepg.br \\ ${ }^{2}$ Institute for Astronomy, University of Hawaii, Woodlawn Dr. 96822, HI, USA \\ email:kuhn@ifa.hawaii.edu \\ ${ }^{3}$ Solar Physics Group, Stanford University, Stanford, CA, 94305, USA \\ email:rbush@solar.stanford.edu
}

\begin{abstract}
In this work we describe the method and results of precise solar astrometry made with the Michelson Doppler Imager (MDI), on board the Solar and Heliospheric Observatory (SOHO), during one complete solar cycle. We measured an upper limit to the solar radius variation, the absolute solar radius value and the solar shape. Our results are 22 mas peakto-peak upper limit for the solar radius variation over the solar cycle, the absolute radius was measured as $959.28 \pm 0.15$ arcsec at $1 \mathrm{AU}$ and the difference between polar and equatorial solar radii in 1997 was $5 \mathrm{~km}$ and about three times larger in 2001 .
\end{abstract}

Keywords. Sun: fundamental parameters, (Sun:) solar-terrestrial relations, Sun: oscillations, Sun: photosphere

\section{Introduction}

The Michelson Doppler Imager (MDI) on board the Solar and Heliospheric Observatory ( $\mathrm{SOHO}$ ) satellite measured the variation and the absolute value of the solar radius and the solar shape (Kuhn et al. 1998, Emilio et al. 2000, Kuhn et al. 2004, Emilio et al. 2007, Kuhn et al. 2009) over a complete solar cycle now.

Several ground-based experiments have been proposed in order to describe the phenomenon of solar radius variations that led to contradictory results. Gilliland (1980) and Laclare et al. (1996) claim to have found a negative correlation when the variation in solar diameter was compared with the solar cycle, while other groups (Ulrich \& Bertello 1995 and Noël 1997) found a direct correlation or no correlation (Emilio \& Leister 2005, Wittman \& Bianda 2000, Brown \& Chistensen-Dalsgaard 1998) at all. By eliminating the effects due the terrestrial atmosphere, the precision for relative variations was found to be better than any previously-reported ground-based measurements.

The problem of determining the solar radius from CCD imagery is, in principle, well defined. In general its solution involves: 1) detecting a fiducial reference height in the solar atmosphere, that for this work is defined from the inflection point in the radial limb darkening function (LDF), and 2) deriving the physical image scale of the optical system. Solar radius measurements are obviously limited when changes in the real or apparent limb profile obscure the fiducial height in the atmosphere. This occurs, for example, if the vertical temperature structure in the solar atmosphere changes or, more importantly, if the point spread function of the optical system varies significantly. The image scale is also affected by perturbations in the optical instrument. The precision of the MDI radius measurements is of order 1 milliarcsec and at this scale several instrumental apparent 
radius fluctuation mechanisms are a concern. These are small effects by the standards of most astronomical imaging experiments, but they can dominate these radius change data.

\section{The MDI Instrument}

MDI is a complex telescope and narrow-band filtergraph instrument designed primarily for measuring the velocity structure in the photosphere (Scherrer et al. 1995). Its effective focal length is $2.18 \mathrm{~m}$ and the CCD detector has $1024 \times 102421 \mu \mathrm{m}$ pixels yielding a nominal pixel scale of $1.96 \mathrm{arcsec} / \mathrm{pixel}$.

A front window protects the internal optics from the space environment and limits the solar energy flux into MDI. It consists of a multilayer dielectric $5 \mathrm{~nm}$ bandpass coating sandwiched between red and yellow Schott glass plates. It transmits about $2 \%$ of the incident solar energy at wavelengths near the Ni I line at $677 \mathrm{~nm}$. A simple refractive telescope using a $12.5 \mathrm{~cm}$ diameter primary lens and a negative enlarging secondary lens is supported by an invar metering structure to maintain a fixed separation against temperature changes.

The light passes through a pair of fixed focus blocks, a selectable polarizer, and a polarizing beamsplitter which sends half the incoming light to a limb sensor for the image stabilization system and the remainder to the filter section. The narrowband filter consists of a $0.8 \mathrm{~nm}$ blocking filter, a Lyot filter, and a pair of Michelson interferometers. Reimaging optics with two selectable light paths produce a final image at the CCD detector of either the full solar disk or a higher resolution patch near disk center.

Figure 1 plots temperatures of the MDI front window, primary lens, and secondary lens over the course of the SOHO mission. The MDI optics package heater adjustments in 1998 November and 2002 February are clearly evident. The annual variation in the temperatures is due to the modulation of the incident power on MDI resulting from the orbital changes in the Sun-to-SOHO distance. The annual peak-to-peak temperature variation of the front window, primary and secondary lenses are $4.5{ }^{\circ} \mathrm{C}, 2.5{ }^{\circ} \mathrm{C}$ and $2.0^{\circ} \mathrm{C}$ respectively. Another important effect is the long term change in MDI temperatures, particularly the front window, due to changes in the instrument thermal properties resulting from long term space exposure.

Most of the internal MDI optics are relatively immune to the small input power variations through the front window, since these are only of order $0.1 \mathrm{~W}$. The only element which sees a large variable radiative thermal load is the primary lens, which is within one $\mathrm{cm}$ of the front window. The window thermal model included this BK7 singlet lens and verified that it does experience a negligible temperature gradient although its mean temperature fluctuates significantly in response to the incident solar flux heating the MDI window. Increasing the temperature of the primary lens by $5{ }^{\circ} \mathrm{C}$ decreases its focal length fractionally by about $3 \times 10^{-5}$ because of the temperature dependence of the BK7 glass. We include this effect in our model of MDI temperature perturbations of the image scale.

Beyond the secondary lens the optical support structure (OSS) is fabricated primarily of aluminum which has a relatively large thermal expansion coefficient. We model temperature-induced OSS expansion by perturbing the lens separations of the elements beyond the invar structure in the ray trace model. As we show below, temperature changes of the OSS do affect the apparent solar radius. 


\section{Solar Radius Variations}

The data and analysis approach used here to measure the radius change is similar to the effort to obtain accurate solar limb shape measurements (Kuhn et al. 1998). The dataset consists of a 6 pixel wide annulus of intensity measurements obtained from solar images produced during the routine operation of the MDI experiment. The 1 minute cadence images are low-pass filtered (to remove solar 5-minute p mode intensity oscillations) and the limb pixels (2"/pixel) are downlinked every 12 minutes.

Each image is first calibrated with a "flat-field" which removes pixel-pixel intensity calibration variations to an accuracy of about $1 \%$. Accurate (to 0.02 arcsec) image centers are derived using an iterative least-squares technique. Limb darkening functions (LDF) are computed from histograms of the pixels in each of 16 angular subregions around the limb. Each 22.5 degree sector yields a LDF for that angular bin within about 6 arcsec of the limb. Figure 2 shows a family of LDFs, $I\left(r, \theta_{i}\right)$, for a typical image. These limb profiles are significantly broader than either the true solar limb or the telescope diffraction limit because MDI images are defocused to minimize aliasing due to the relatively large (2 arcsec) pixels.

We define the limb edge position by least-squares fitting a gaussian plus quadratic background function of the radial distance from image center, $r$, to the square of the radial derivative of the mean LDF. The squared LDF ensures positive data values even in the case of large noise fluctuations. These data are also indicated in Figure 3 along with the derived fit to this profile. We adopt the maximum of this fitting function as the limb radius. Low order optical aberrations are effectively eliminated by averaging the radial limb position around the limb, over the 16 angular bins.

Except for the early data period the LDF appears relatively stable with shape fluctuations (Kuhn et al. 2004) which bias the derived radius changes by much less than

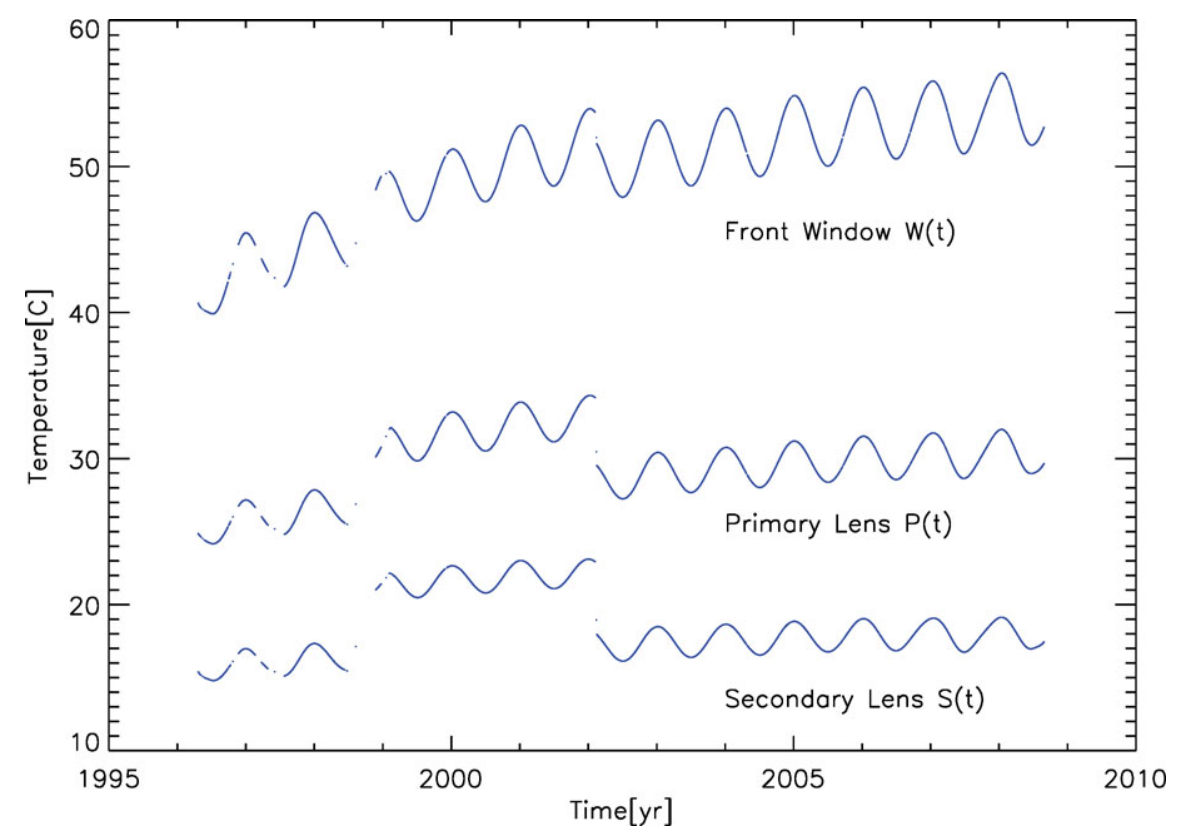

Figure 1. Recorded temperature of the MDI primary lens, front-window mounting ring and secondary lens mount as a measure of the OSS is plotted. 
0.01 pixel. Errors in the early $\mathrm{FB}=1$ data were as large as 0.01 pix and we discard these in the following analysis.

Periodically we empirically determined the best MDI focus. This was derived from solar images obtained at each focus block setting. The intensity contrast from each image was determined and averaged over a region near the image center. The best fractional focus block setting was then obtained by interpolating the integer domain empirical dependence of contrast on focus block setting to find the maximum contrast and best focus condition.

Each of these thermally induced perturbations affect the final image scale and the best focus of the instrument. We compute the change in the apparent solar radius from our ZEMAX ray trace model by computing how the middle of the blur circle of a field point at an angle of $0.267^{\circ}$ (approximately one solar radius) is shifted. The best focus is obtained by computing the rms blur radius at each focus block (FB) optical configuration and then interpolating to the fractional $\mathrm{FB}$ value which minimizes the blur diameter. The model optical configuration is symmetric about the optic axis so that this calculation can only depend on the radial field angle.

The accuracy of these corrections is limited by our knowledge of the precise form of the temperature perturbations. For example, we do not know the true temperature gradients in the MDI OSS, nor do we know the actual temperature distribution across the front window. Based on our knowledge of the model parameters and experience with the perturbed optical system we believe the OSS expansion term is accurate to about $50 \%$, while the primary lens perturbation is accurate to $10 \%$. Thus, the window gradient term is particularly sensitive to the absolute instrument focus condition, which is relatively weakly determined. The gradient sensitivity we calculate here is consistent with the MDI optics model but could change by a factor of two within our parametric model uncertainties. Here we refer to the "accuracy" of a perturbation as the fractional uncertainty in derived focus and radius changes caused by a given temperature change.

Figure 4 indicates that an accurate solar radius determination must rely on understanding and correcting for systematic radius noise sources and that the instrument temperature perturbations clearly dominate these systematics. Figure 1 showed that the window temperature, primary lens, and secondary temperatures vary in phase with the

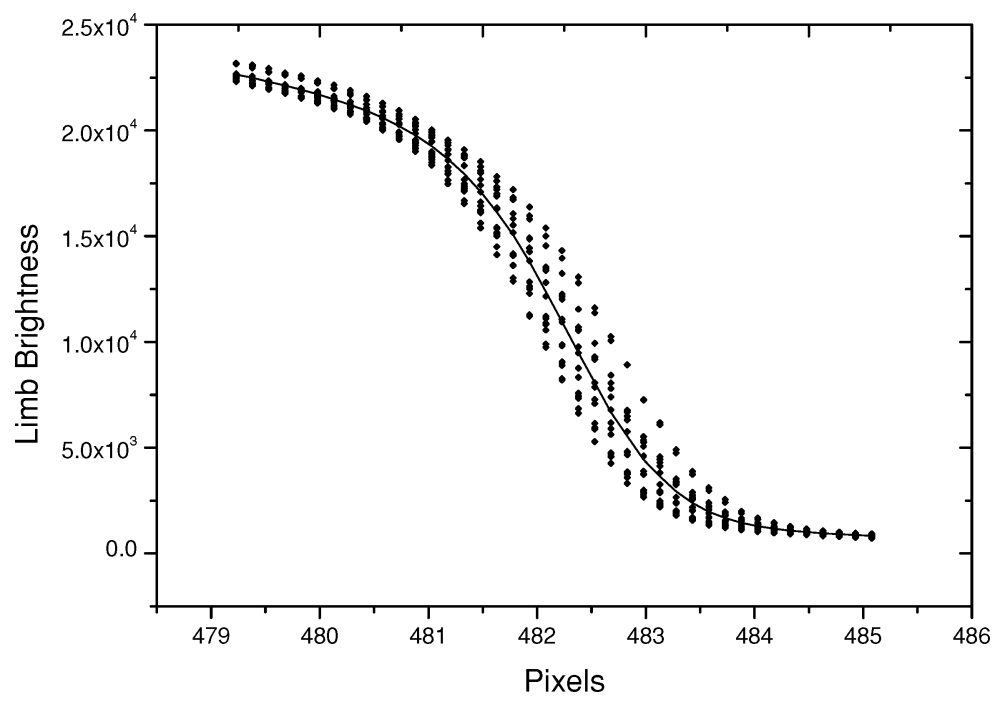

Figure 2. This figure shows a family of limb darkening functions (LDF's), $I\left(r, \theta_{i}\right)$, for a typical image. Each $22^{\circ} .5$ sector yelds a LDF for that angular bin within about 6 " of the limb. 
yearly apparent radius change of 0.2 arcsec-AU in Figure 4 . In our discussion below we use the secondary lens measurements as a proxy for the average OSS temperature.

Important features of the MDI temperature record are: 1) the yearly variation due to the changing incident solar heat flux, 2) the front window opening transient response near the end of 1998 and, 3) the deliberate change in MDI heater power levels in 1998 and 2002. These temperature perturbations can be directly related to the apparent radius and focus changes using the optical perturbation model.

An important check of the thermal response model comes from a calculation of the a priori apparent solar radius changes caused by the yearly fluctuations in the window temperature gradient, the primary lens temperature, and OSS structure temperature. Thus, using empirical yearly temperature changes and the apparent radius sensitivities computed, we calculate a radius change of 0.1 pixel and focus change of $-0.1 \mathrm{FB}$ that are synchronous with the annual variation in solar heating. Given the systematic errors in the three components which add and subtract to yield it, it may be fortuitous that this expected change of 0.1 pixel is in such good agreement with the observed 0.1 pixel $(0.2$ arcsec-AU) annual variation. Evidently our simplified thermal model is consistent with the observed annual MDI fluctuations.

The transient response of MDI, after opening the front window and allowing solar radiation to enter the instrument in late 1998, is expanded in Figure 5. From the thermal model of the front window we've also plotted the edge-to-center temperature difference versus time. Figure 5 also shows the derived best focus position and the change in the observed solar radius (at focus block 4). The data obtained from MDI as it responded to this relatively large thermal transient allow us to quantitatively test the different thermal contributions to the optical variability of the best focus and apparent radius.

The front window eventually heats by $28^{\circ} \mathrm{C}$, and after 95 minutes has risen in temperature to $80 \%$ of this maximum. According to the finite element thermal model, the

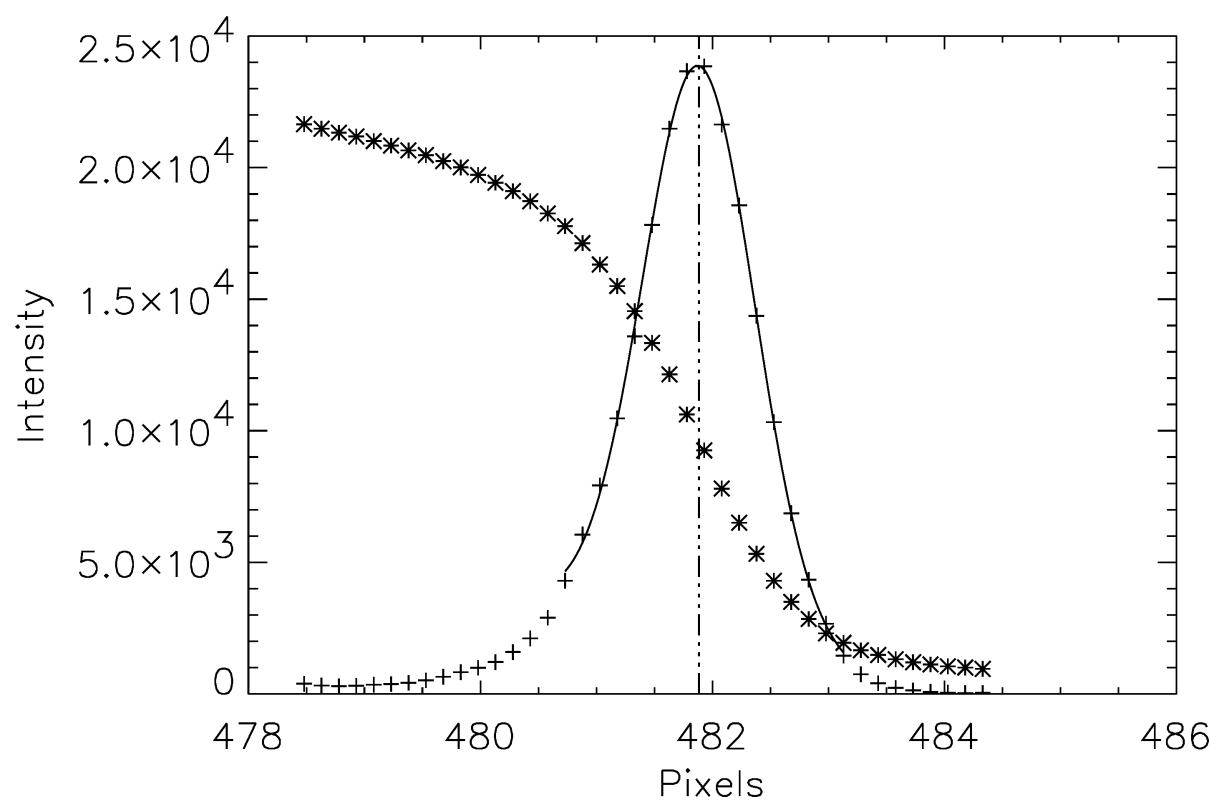

Figure 3. Typical limb-darkening function data from a single observation. The limb intensity is plotted with star symbols. The squared radial derivative is shown with plus sysmbols, and the curve shows the derived fit whose maximum value determines the solar radius. 
window temperature gradient reaches a maximum of about $4{ }^{\circ} \mathrm{C}$ after 40 minutes, while after only 15 minutes it is at $80 \%$ of this maximum. The primary lens heats by $13{ }^{\circ} \mathrm{C}$ but achieves $40 \%$ of this after 95 minutes. The secondary lens has an even smaller and slower response, increasing by $8{ }^{\circ} \mathrm{C}$ and only $25 \%$ of this maximum after 95 minutes.

The best focus setting reaches a minimum after only 40 minutes and declines by 3.7 focus blocks. It then increases by $0.8 \mathrm{FB}$ after 95 minutes. The apparent radius increases by 0.3 pixel over this short period, then decreases by 0.05 pixel between 40 and 95 minutes after opening the front window aperture cover.

Qualitatively these results are consistent with the temperature perturbations of our model. The window gradient dominates the focus and radius changes during the first 45 minutes. According to the model a gradient of $4{ }^{\circ} \mathrm{C}$ should decrease focus by $3 \mathrm{FB}$ but increase the apparent radius by 0.3 pixel as it does. This result is in, perhaps fortuitous, agreement with the observed radius change. Nevertheless the window gradient is the only effect which accounts for the large focus shift and apparent radius increase over this short duration.

As part of monthly calibration observations, MDI obtains solar images over a range of focus blocks in order to determine the instrument best focus. Figure 6 plots the best focus over the MDI history. While these data are noisy, a hint of a yearly focus variation around the trend $F(t)$ is apparent and described by a quadratic function.

Encouraged by our success in describing the apparent radius changes caused by variable thermal effects we attempt a "non-statistical" (a priori) direct correction for the radius timeseries by adjusting, and scaling the known temperature perturbations using the model temperature sensitivities. Because the temporal linear increasing trend in the front window temperature does not directly imply an increasing window radial gradient we fit and remove this from the window timeseries data. We already showed that it has an insignificant effect on the OSS expansion and primary lens focal length changes so, for simplicity, we also remove this trend term from the other temperature records.

As we argued above, the internal optics heater changes do not affect the window gradient so we also remove this jump from the window temperature record. This corrected

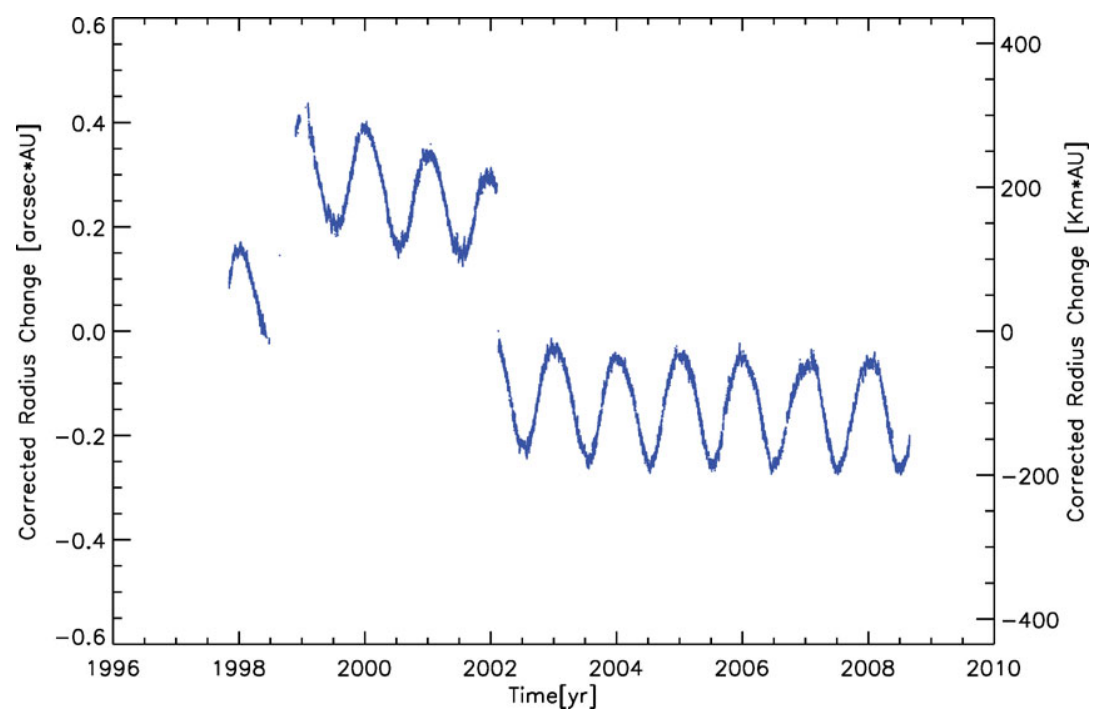

Figure 4. Residual apparent solar radius changes are plotted here after correcting for focus block changes in the instrument to an effective observing distance of $1 \mathrm{AU}$. 
window temperature, $W(t)$, is then a proxy for apparent radius changes due to the annual window gradient variations when scaled by 0.01 pixel $/{ }^{\circ} \mathrm{C}$. Our previous results (Kuhn et al. 2004) for the a priori model we scaled the primary lens temperature, $P(t)$ by -0.009 pixel $/{ }^{\circ} \mathrm{C}$ and the gradient-adjusted secondary lens (OSS) temperature, $S(t)$, scales by $0.038 \mathrm{pixel} /{ }^{\circ} \mathrm{C}$. The final gradient correction comes from the linear trend in best focus from Figure 6. As we argued above, this is due to the decreasing radial window gradient from the increasing front window opacity.

This final correction to the apparent radius timeseries $\delta r(t)$, comes from scaling the linear trend in focus, $F(t)$, by $-0.097 \mathrm{pixel} / \mathrm{FB}$.

Recognizing that our a priori temperature sensitivities are based on an imperfect model we have also allowed the scaling coefficients to vary in a least-squares fitting procedure which minimizes the deviation between the radius variation data and the sum of the scaled temperature proxy functions. Thus we seek a solution for coefficients $w, p$, $s$, and $f$ that minimizes $\sum_{t}(d r(t)-w W A(t)-p P(t)-s S(t)-f F(t))^{2}$. Some of these functions are strongly correlated and in order to constrain the solution and decrease the number of free parameters we fix $w=0.01$ pixel $/{ }^{\circ} \mathrm{C}$ to the a priori value and allow only three fitting variables.

We obtain values for sensitivity parameters of $p=-0.011 \pm 0.002, s=0.041 \pm 0.002$ pixel $/{ }^{\circ} \mathrm{C}$ and $f=-0.079 \pm 0.005 \mathrm{pixel} / \mathrm{FB}$. Those values are different from our previous statistical model in Kuhn et al (2004). Here we obtained the coefficients from the full solar cycle dataset. The coefficients are consistent with our physical model and their expected uncertainties. The residual radius variation obtained after subtracting the bestfit function is plotted in Figure 7.

The data with fitted corrections in Figure 7 are insensitive to any real secular solar radius variability because they are degenerate with the fitted secular trend in the window

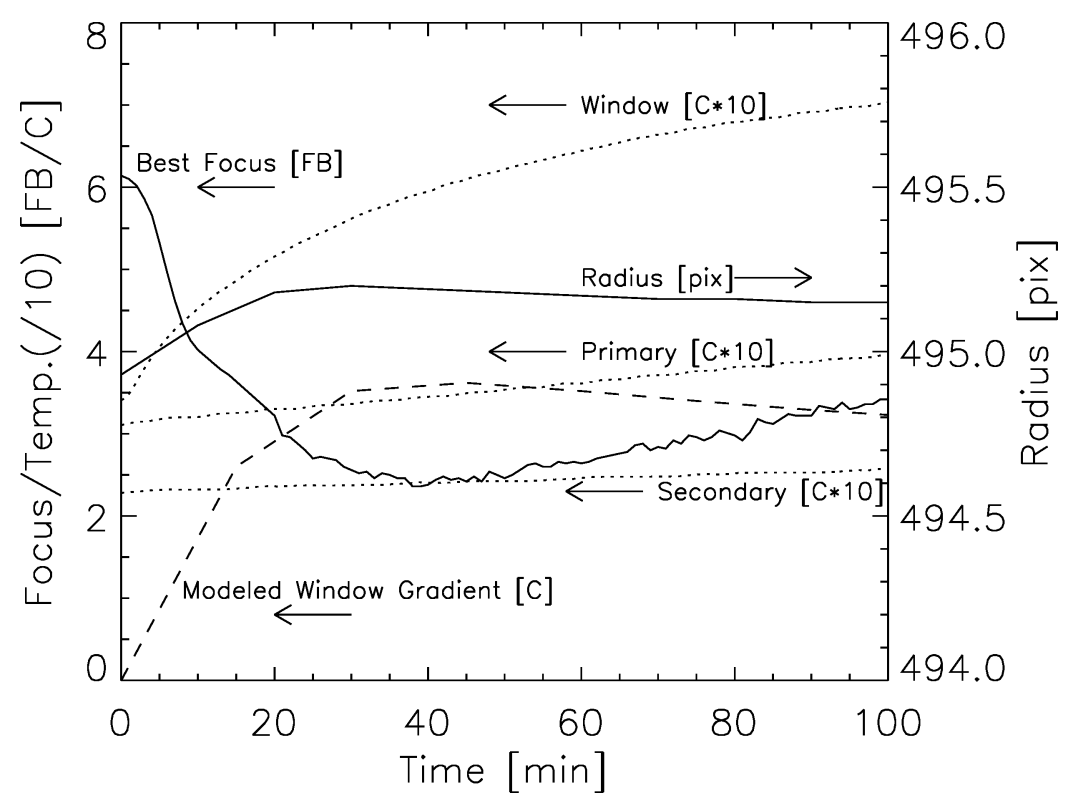

Figure 5. The transient thermal and optical response of MDI after the front window was opened 1998 November 20. Time is measured along the horizontal axis in minutes after the instrument is exposed to sunlight. The best-focus setting in focus block units, the modeled window gradient (in ${ }^{\circ} \mathrm{C}$ ), the temperatures of the window, primary and secondary lens (in 10's of ${ }^{\circ} \mathrm{C}$ ) are indicated on the left. The apparent solar radius (in pixels) is shown on the right vertical scale. 
gradient correction. While we believe the trend in the radius variation data is only caused by the imperfect thermal model, we use these residuals to derive a conservative upper limit to any secular solar radius change. The best-fit line to these data suggests that any linear trend in the solar radius must be smaller than $0.9 \pm 1.6$ milliarcsec/year. The uncertainty were calculated using the bootstrap procedure. This is not a statistically significant trend and implies a $2 \sigma$ upper limit of 3.2 mas/yr or 0.3 arcsec/century. In comparison Shapiro (1980) obtained the same formal upper limit from about 200 years of Mercury transit data.

After scaling the sunspot number solar cycle variation to unity range we have fit this function to the residual radius data using the bootstrap procure. The derived solar cycle radius variation in the statistical corrected data is $-17 \pm 11$ mas peak-to-peak over a solar cycle. This result, which is not statistical significant implies a $2 \sigma$ limit on the amplitude of the solar cycle of 22 mas.

\section{Absolute Solar Radius Determination}

The image scale has been determined from the May 72003 Mercury transit data separately for each focus between FB 3 and 6 (Kuhn et al. 2004). Each of these absolute image scale measurements has a statistical uncertainty of less than $0.0001 \mathrm{arcsec} / \mathrm{pixel}$. In contrast the ray trace model and solar limb data provide accurate measurements of the differential scale change between focus settings. For the solar limb data obtained at focus 3 and 4 (essentially all of the useful radius data) these changes imply an uncertainty in the differential image scale of about $2 \times 10^{-5}$ arcsec/pixel, which is somewhat better than the fractional absolute scale determination uncertainty.

We compute the solar radius from the limb average of the MDI image radius. These data must be corrected for the average radial image distortion which causes the Sun to appear larger than it is. This amounts to $0.92 \pm 0.05$ pixel where 0.32 pixel results

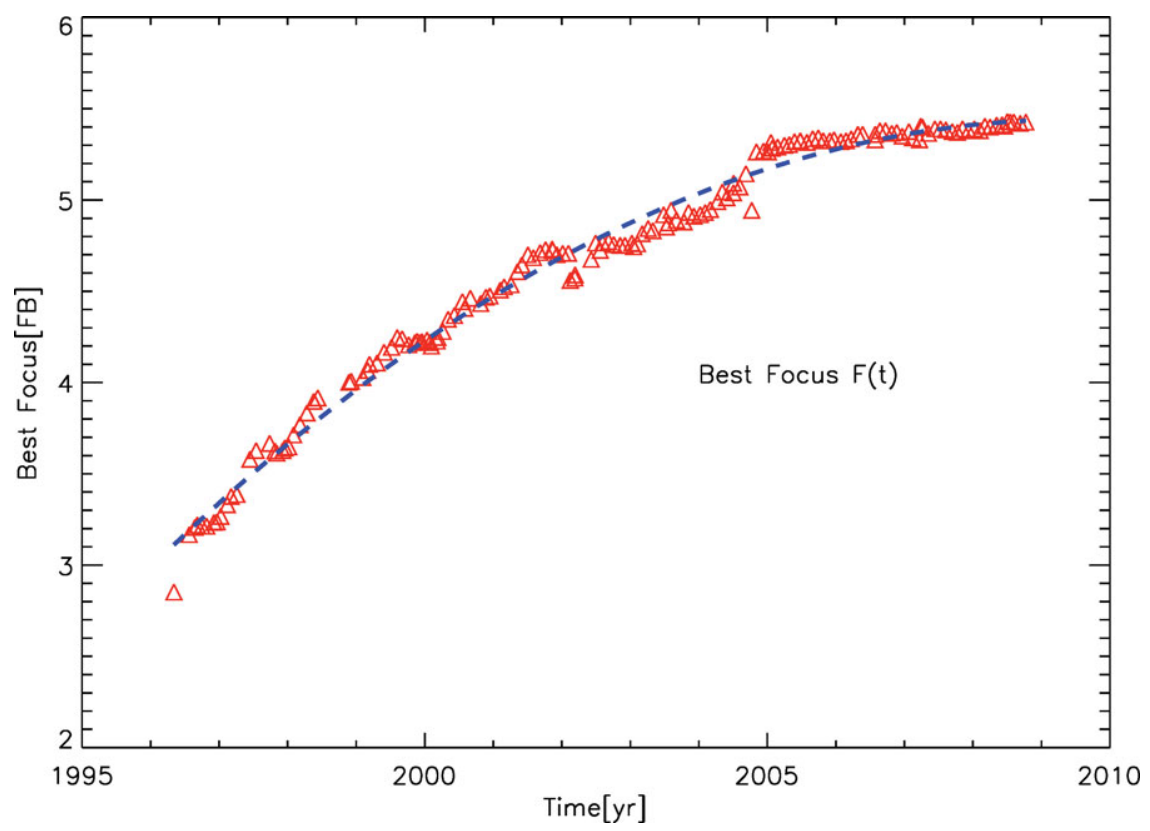

Figure 6. Change in the best-focus in units of focus blocks. The quadratic temporal trend is plotted as the dashed line. 
from image tilt and 0.6 pixel comes from the symmetric image distortion. The distortion uncertainty quoted here comes from the $70 \%$ systematic uncertainty limits in the transit data fit to the model distortion function.

Immediately before and after 2003 May 7, MDI was operating with FB 4. The mean solar image radius near this time was $484.371 \pm 0.0015$ pixel. Correcting for distortion we obtain $483.43 \pm 0.05$ pixel. With FB 4 calibration of $1.9870 \pm 0.0002 \mathrm{arcsec} / \mathrm{pixel}$ we derive a solar radius of $960.58 \pm 0.15$ arcsec (as seen from SOHO) where our quoted error includes the statistical, distortion, and calibration uncertainties. During the transit SOHO was 0.99865 AU from the Sun, so that the apparent solar radius at $1 \mathrm{AU}$ is $959.28 \pm 0.15$ arcsec and the error reflects all known statistical and systematic uncertainties.

\section{Solar Shape}

Small changes in the form of the local LDF, $L(r, \theta)$, at solar colatitude $\theta$, contain solar shape and radius information. We measure $\theta$ in the detector array coordinates, but $\mathrm{SOHO} / \mathrm{MDI}$ keeps the projected rotation axis of the sun accurately lined with the vertical axis of the array. Distinguishing the raw shape signal, $\beta(\theta)$ - the radially displaced mean LDF defined by $L(r-\beta(\theta))$ - from other solar and instrumental perturbations to $L(r, \theta)$ is non-trivial. This is because the solar shape is typically 2 to 3 orders of magnitude smaller than the LDF measurement pixel size (approximately 2 arcsec in MDI) and about the same factor smaller than the diffraction limit of the telescope. At this level, small latitudinal variations in the solar atmosphere and instrumental effects can contribute to the observed LDF and the apparent shape signal.

Full-disk images were obtained in 2001 as they were in 1997 by stepping SOHO by 30 degree increments through 360 degrees in roll angle. At each position approximately 5 MDI continuum filtergram images were obtained with the MDI fast tip-tilt steering mirror engaged. These images were reduced to measurements of the local LDF in the

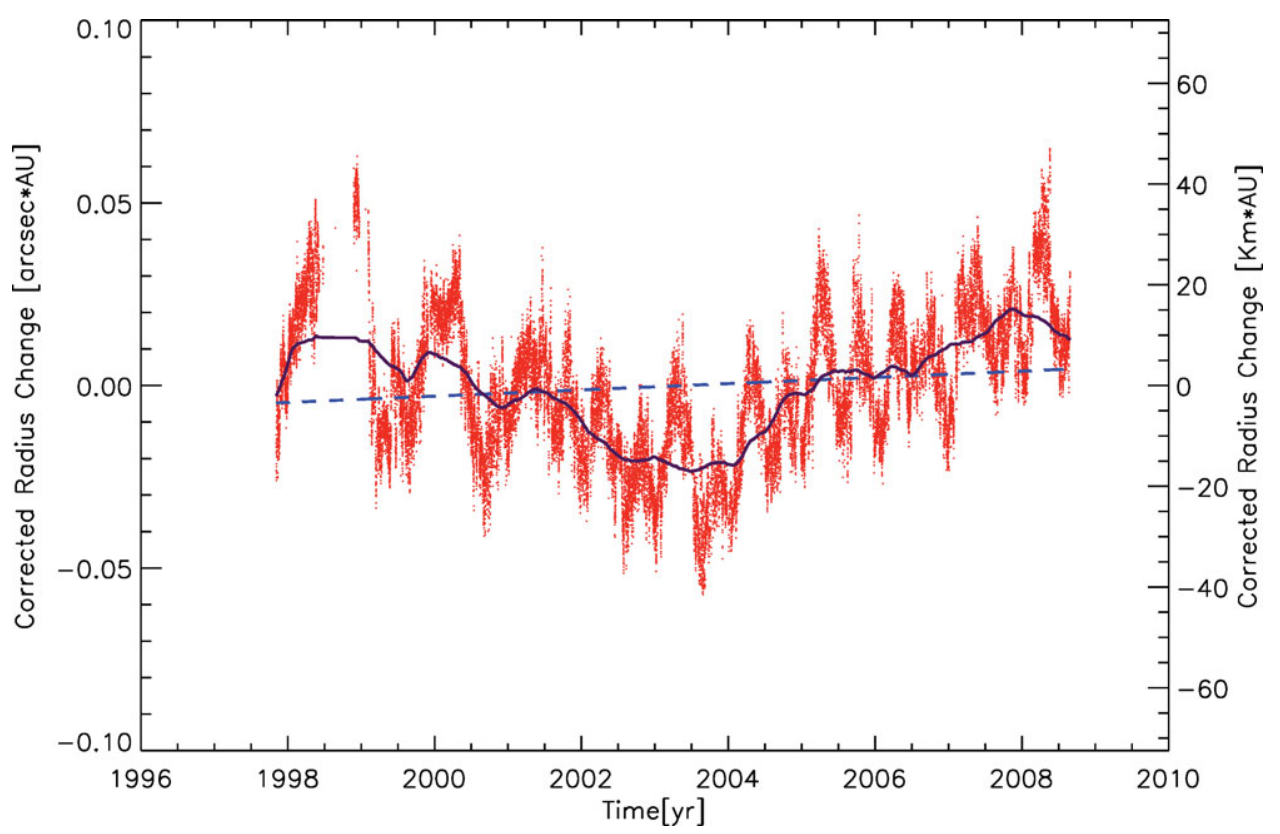

Figure 7. Residual variation of the statistical least-square model. 
same way 1997 observations were analyzed except that the data were binned into 360,1 degree angular bins.

In 1997 we also suppressed instrumental effects by rotating the spacecraft. The derived shape signal $\beta=\beta_{s}+\beta_{i}$ consists of slow spatially varying instrumental $\left(\beta_{i}\right)$ and $\operatorname{solar}\left(\beta_{s}\right)$ contributions. The stability of the instrument during the satellite roll procedure allows us to recover $\beta_{s}$ even when it is much smaller than $\beta_{i}$. We modify our 1997 analysis to account for localized shape function contamination from active regions near the limb. We also improved the determination of the mean LDF used as the fitting functions in our least-squares fit for $\alpha, \beta$ and $\gamma$ parameters. The previous analysis used the first order LDF which was smeared by the instrumentally induced limb shape distortion. In this work we corrected this effect by measuring the $r$ shift of the smeared $L(r)$ with relation to $L(r, \theta)$. These shifts, $\beta(\theta)$, are then used to shift $L(r-\beta(\theta), \theta)$ and then average over $\theta$ to compute an unsmeared LDF.

Our model for the apparent LDF is

$$
L(r, \theta)=(1-\alpha(\theta)) L\left(r-\beta_{s}(\theta)-\beta_{i}(\theta)-F(\theta)\right)+\gamma(\theta) L^{\prime \prime}(r)
$$

In practice we made a least square fit using our empirical (unsmeared) LDF function $L(r)$ and its first and second derivatives as fitting functions. The corresponding coefficients $1-\alpha(\theta), \beta(\theta)$ and $\gamma(\theta)$ are found for each roll angle. Initially $F(\theta)$ is taken to be zero, but we redo this fit using the $\alpha(\theta)$ obtained from the previous iteration with $F=f \alpha$ (the constant $f$ is described below). The solar limb shape function, $\beta_{s}$ is recovered, as it was in 1997, from the sequence of 12 rotated LDF datasets using a phase modulation

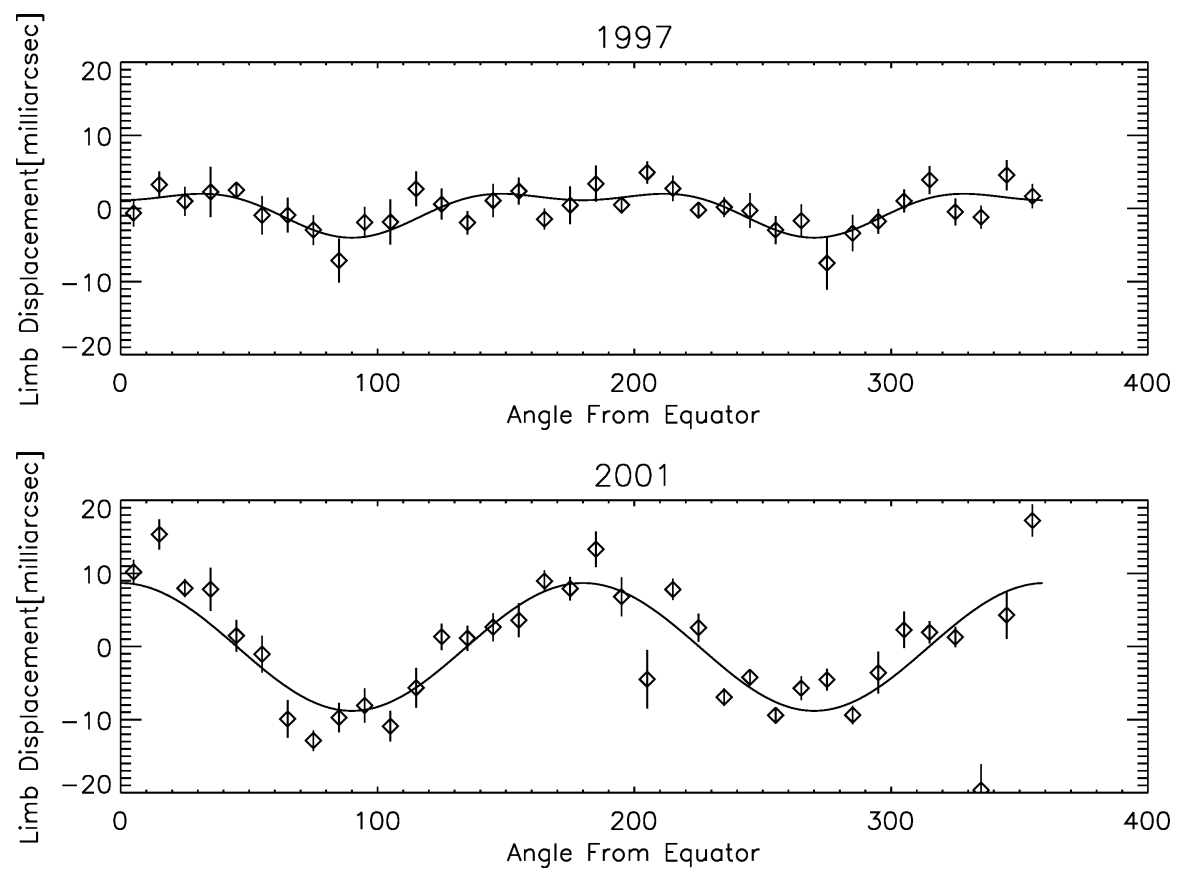

Figure 8. The limb shape from 1997 and 2001 is plotted here. The equator corresponds to position angle 0 . Data have been averaged into 10 degree wide bins and the best fit $l=2$ (oblateness) and $l=4$ (hexadecapole) Legendre Polynomial have been plotted. The shape distortion after correcting for bright contamination is nearly a pure oblateness term in 2001, while 1997 has a significant hexadecapolar shape contribution. 
approach (Kuhn et al. 1998) This method averages azimuthal fourier transforms of the $\beta(\theta)$ transforms from each roll angle to separate $\beta_{i}$ from $\beta_{s}$.

Figure 8 shows how the faculae-corrected limb shape changed from 1997 to 2001 (Emilio et al. 2007). The solid curve in each graph is a fit to a quadrupole and hexadecapole shape distortion. Our previous analysis of the 1997 data (Kuhn et al. 1998) yielded marginally different (at the 2- $\sigma$ level) results. That analysis did not use all of the image stabilized limb observations from the spacecraft and it did not include fitting terms for the known tilted-CCD instrumental effect in the MDI detector. The binned data in figure 2 shows a difference between the equatorial and polar radii in 1997 of $8.7 \pm 2.8$ milliarcsec and $18.9 \pm 1.9$ milliarcsec in 2001. Least squares fits of a second and fourth order legendre polynomial give a solar shape that is nearly purely oblate near solar maximum, but has a significant hexadecapole shape near minimum. The oblateness shape term changed from $-3.1 \pm 0.7$ to $-12 \pm 2$ milliarcsec while the hexadecapole went from $-1.7 \pm 0.9$ to $0.3 \pm 2.5$ milliarcsec from 1997 to 2001.

\section{Conclusion}

The SOHO MDI experiment has yielded accurate measurements of the solar radius and radius variability from above the Earth's atmosphere. We have shown that it is possible to determine the temperature sensitivity and optical characteristics of MDI with sufficient accuracy so that a statistical calibration of instrumental systematics is not necessary. The limit MDI achieves on possible solar cycle radius variations is 22 milliarcsec.

This is the first accurate optical solar radius measurement from above the atmosphere. The derived radius is precisely defined with respect to the inflection point in the unblurred solar limb darkening profile. Adopting the value $1 \mathrm{AU}=1.495979 \times 10^{8} \mathrm{~km}$ we find the Sun's radius to be $R_{\odot}=6.9574 \pm 0.0011 \times 10^{5} \mathrm{~km}$. This is slightly smaller than recent ground-based measurements (Brown and Christensen-Dalsgaard 1998) but is consistent with the highly precise helioseismic determination (Schou et al. 1997) of $6.9568 \pm 0.0003 \times 10^{5} \mathrm{~km}$. The fundamental limitation to our measurement uncertainty comes from incomplete knowledge of the MDI optical distortion. Our limits on solar radius variability are consistent with earlier precise MDI and helioseismic measurements (Emilio et al. 2000 and Dziembowski et al. 2001) but are established here from image data without assumptions about systematic temporal instrument variations.

A significant discrepancy between ground-based observations and these results is clear. The ensemble of solar radius and variability data from the ground instruments suggests that they may be seeing effects caused by the terrestrial atmosphere and potentially solar cycle induced variability in the Earth's atmosphere. This may explain the apparent solar radius variations with instrument latitude and phase during the solar cycle, although this remains an important issue to be resolved.

The difference between polar and equatorial solar radii in 1997 was $5 \mathrm{~km}$ and it was about 3 times larger than this in 2001. While these are highly statistically significant values they are tiny in comparison to physically interesting length scales in the photosphere. For example the density scale height is $150 \mathrm{~km}$ and the smallest (granulation) convection scale near the photosphere is $700 \mathrm{~km}$.

\section{Acknowledgements}

This research was supported by the NASA SOHO/MDI grant NNX07AK36G to Stanford University and the NASA SDO/Helioseismic and Magnetic Imager contract NAS502139 to Stanford University and subcontract to the University of Hawaii. 


\section{References}

Brown, T. M. \& Christensen-Dalsgarrd, J. 1998, ApJ, 500, L195

Dziembowski, W. A., Goode, P. R., Kosovichev, A. J., \& Schou, J. 2001, ApJ, 553, 897

Emilio, M., Kuhn, J. R., Bush, R. I., \& Scherrer, P. 2000, ApJ, 543, 1007

Emilio, M. \& Leister, N. V. 2005, MNRAS, 361, 1005

Emilio, M., Bush, R. I., Kuhn, J. R., \& Scherrer, P. 2007, ApJ, 660, L161

Gilliland, R. L. 1980, ApJ, 248, 1144

Kuhn, J. R., Bush, R. I., Scherrer, P., \& Scheick, X. 1998, Nature, 392, 155

Kuhn, J. R., Bush, R. I., Emilio, M., \& Scherrer, P. H. 2004, ApJ, 613, 1241

Kuhn, J. R., Emilio, M., \& Bush, R. I. 2009, Science, 324, 1143-b

Laclare, F., Delmas, C., Coin, J. P., \& Irbah A. 1996, Solar Phys., 166, 211

Wittmann, A. D. \& Bianda, M. 2000, In: Proceedings of the 1st Solar and Space Weather Euroconference, ESA SP 463, 113

Scherrer, P. H., Bogart, R. S., Bush, R. I., Hoeksema, J. T., Kosovichev, A. G., Schou, J., Rosenberg, W., Springer, L., Tarbell, T. D., Title, A., Wolfson, C. J., Zayer, I., \& MDI Engineering Team 1995, Solar Phys., 162, 129

Schou, J., Kosovichev, A. G., Goode, P. R., \& Dziembowski, W. A. 1997, ApJ, 489, 197

Shapiro, I. I. 1980, Science, 208, 51.

Ulrich, R. K. \& Bertello, L. 1995, Nature, 377, 214 